\title{
Las analogías en la enseñanza y el aprendizaje de las ciencias experimentales: un estado de la cuestión
}

The analogies in the teaching and learning of experimental sciences: A review of literature

Natalia Buitrago Henao y Ana Maŕa Wamba

nbuitrago@ayura.udea.edu.co, muamba@uhu.es

\section{Resumen}

Esta ponencia hace parte del trabajo final para optar al título de Máster Oficial en investigación en la enseñanza y el aprendizaje de las ciencias experimentales, sociales y matemáticas, de la Universidad Internacional de Andalucía; y, presenta un estado actual de la cuestión en torno al uso de las analogías como recurso didáctico en el aula de ciencias.

Palabras clave

Analogías, Enseñanza y Aprendizaje de las Ciencias, Modelización, Desarrollo Profesional del Profesorado.

\section{Summary}

This paper does partof the final work to choose to Master degree title in research in the education and the learning of the sciences experimental, sciences social and mathematical, of the International University of Andalusia; and, he presents a current condition of the question around the use of the analogies as didactic resource in the classroom of sciences.

\section{Key words}

Analogies, Education and Learning of the Sciences, Modeling, Professional Development of the Professorship.

\section{Introducción}

Para el desarrollo de esta investigación se revisaron diversos artículos que relacionan las analogías con la enseñanza y el aprendizaje de las ciencias experimentales; los cuales demostraron varias perspectivas de investigación en el uso de las analogías, que en este artículo, se dividen en los siguientes aspectos: a) Desarrollo profesional del profesorado -en tomo a su práctica, epistemología-; b) Aprendizaje por medio de analogías como recurso didáctico -aula y libros de texto-; c) Modelos didácticos para la enseñanza de las analogías. A continuación se hace una descripción de los estudios y de las condusiones que estos reporta.

\section{Desarrollo profesional del profesorado}

En el desarrollo profesional del profesorado, el conocimiento del contenido, el conocimiento didáctico del contenido, y el conocimiento pedagógico, (en el sentido que lo define Porlán en sus teorías) juega un papel relevante a la hora de comprender el uso que hacen los maestros de las analogías. Un número significativo de investigaciones y avances en esta línea, se debe a los aportes 
de José María Oliva y col. (2003, 2004, 2005, 2007). Oliva et.al. (2001) realizan una investigación en la cual ponen de manifiesto las concepciones y creencias de 37 profesores de ciencias en activo de Educación Secundaria participantes en un curso de formación. Con los datos aportados, los autores conduyen que las confusiones sobre lo que es una analogía tiene un origen de tipo semánticas; confusiones que fueron superadas luego de asistir al curso. Además, manifiestan la restricción del uso de las analogías solo cuando esta implica una relación en un sentido irreal o figurativo; concepciones que evolucionaron sin mayor dificultad. Así mismo la mayoría de los docentes solían ser conscientes de las ventajas e inconvenientes que planteaba el uso de analogías; si bien los métodos didácticos que declaraban utilizar en sus dases no parećan los más idóneos ni adecuados para superar esas dificultades. Esta contradicción puede explicarse si tenemos en cuenta que el profesorado mantiene una serie de creencias implícitas que son más relevantes de cara a dirigir su actividad práctica que las propias ideas o nociones que manifiestan verbalmente. Sobre este punto, en esta investigación se identificaron tres creencias implícitas en tomo a las analogías como estrategia de enseñanza, que son: en primer lugar, los inconvenientes de las analogías tienen que ver con su uso como herramienta didáctica y con las características del alumno, más no con los métodos de enseñanza a través de los cuales se plantea su uso; en segundo lugar, la analogía consiste en un "artefacto" que se aprende y no en un proceso, y por último al enfrentarse a una analogía los alumnos se comportan como una tabula rasa completando huecos en su memoria con los contenidos que le transmite el profesor. Estas creencias evolucionaron muy poco o no sufrieron modificaciones lo cual amerita investigaciones posteriores.

En Oliva (2003) se presentan otros aspectos de este estudio. Al analizar los datos es conduyente que el profesorado participante en la investigación valora las analogías como un recurso útil para la enseñanza y reconoce así mismo, hacer un uso frecuente de ellas en sus dases. En el caso del profesorado de Física y Química, los temas que más parecen prestarse al uso de analogías son los relacionados con la estructura atómica y el enlace químico, la electricidad y la comiente eléctrica. De la perspectiva del uso de las analogías en el aula, según el autor se puede identificar un guión general estableciendo algunos rasgos. Las analogías surgen normalmente durante las explicaciones o durante la preparación de las dases. Se introducen preferentemente al comenzar el tema o de forma continuada a lo largo de todo el mismo. Se presentan por vía de transmisión oral del profesor al estudiante. El profesor trata de ejercer un cierto control sobre el grado de comprensión que los estudiantes adquieren de la analogía: corregir errores y analizar similitudes. Este modelo permite inferir una perspectiva didáctica ubicada a medio camino entre un modelo tradicional y un modelo tecnológico de enseñanza y se muestra coherente con las concepciones y creencias que mantiene el profesorado hacia las analogías como recurso en el aula, en plena correspondencia con sus teorías implícitas, el autor afirma en este punto, que un aspecto importante de los programas de formación de profesores es la necesidad de configurarse para atender a diferentes comportamientos y creencias de los participantes. Posteriormente Oliva (2004) presenta planteamientos respecto a las características del razonamiento analógico. Además, de confrontarlos con algunas de las concepciones y creencias que según el autor suelen mantener los profesores de ciencia sobre el razonamiento analógico. Se plantea al carácter interno que tiene la construcción de la analogía como fenómeno asociado al estudiante y no sólo a la explicación del profesor.

Además, se sugiere la naturaleza procesual de la analogía como fenómeno de transferencia de significados entre dominios. Adicional a estos argumentos, se resalta la importancia del modelo sobre el que descansa la analogía, como mediador del proceso de transferencia analógica y como lugar de encuentro desde el que interpretar la intencionalidad y finalidad de la analogía. También se plantea, el carácter complejo del proceso de transferencia analógica a través del modelo de la analogía, como fenómeno bidireccional e interactivo.

Anexo a lo anterior, se resalta el valor de las analogías en el desarrollo de procedimientos y actitudes científicas en los estudiantes basado en el aprendizaje como proceso de construcción 
personal e interpersonal. En consecuencia, Oliva, Azcarate y Navarrete (2007) plantean un estudio que al analizar los datos se conduye que la mayoría de los profesores correspondían a modelos tradicionales para el uso de las analogías o en el mejor de los casos, a modelos basadas en un aprendizaje significativo por la transmisión / recepción de aclarar antes conocimientos. Así mismo Oliva (2005) como parte de otro estudio, presenta un análisis de los conocimientos deseables de los profesores de física para hacer uso de analogías como un recurso educativo. Según el autor, los docentes deben de tener tanto los conocimiento conceptuales como las destrezas para manejarlos y una práctica coherente, dado que una analogía es una construcción personal donde el profesor debe guiar al estudiante interactuando con él, con el fin de que el estudiante elabore su propio modelo.

Oliva (2006) plantea en un primer momento, la importancia de la participación activa del estudiante en el uso de analogías como recurso de enseñanza en la dase de la Química. En un segundo momento, se discute la importancia de la labor del profesor en este contexto, como agente necesario para guiar el proceso de elaboración de la analogía que siguen los estudiantes. Adicional a esto, plantea la reelaboración en cuadros que permitan visualizar la trama de nexos y las limitaciones 0 , la presentación en lecturas que planteen orientación comprensiva de las analogías; estas herramientas buscan promover y favorecer el pensamiento analógico de sus estudiantes, ya que les permite según el autor, aportar ideas y tomar decisiones en cuanto a qué analogía desean construir, aunque dentro de ciertos límites y variedad de opciones a través de una labor orientada y guiada por el profesor.

\section{Aprendizaje por medio de analogías}

Uno de las mayores producciones en lo que respecta al análisis del uso adeauado de las analogías en los libros de ciencias lo encontramos en Femández et al. (2000) quienes en un primer lugar, realizan una revisión bibliográfica de los modelos de enseñanza con analogías y plantea criterios con la finalidad de detectar y dasificar la naturaleza de las analogías presentes en las siete editoriales que daría con el hallazgo de 339 analogías, encontradas en 84 libros de texto aryo análisis arrojan los siguientes resultados bajo los criterios que los autores proponen en el texto encuentran que el tratamiento de las analogías es pobre e inadecuado. Se reporta también, que los editores de los textos no tienen en cuenta a las analogías como una herramienta de aprendizaje y en caso de abordarlas se olvidan de que su potencial depende de que los estudiantes comprendan el análogo a fondo; además de identificar la estrategia analógica y las correspondencias entre el análogo y el tópico. Este proceso de comprensión, identificación, extracción y transferencia de similitudes deber ser suministrado por el autor del libro de texto. En otro estudio de Femández et al. (2003) se afirma que el uso de las analogías en los libros de texto se hace de una manera poco reflexiva y estudiada. De igual forma, afirman que se conoce poco del proceso cognitivo de aprendizaje a través de la modelización con analogías. Conocer el razonamiento analógico de los estudiantes y de los profesores es fundamental en el campo de las Ciencias, por cuanto ésta trabaja de forma generalizada con modelos conceptuales, modelos de procedimiento, modelos mentales, concepciones altemativas.

En un artículo adicional (Femández et al. 2005 a) además presenta la compleja relación que existe entre modelo mental y modelización, para acercarse a una definición de analogía, que induya las semejanzas estructurales y las semejanzas superficiales. Adicionalmente, presenta un consolidado para conocer la distribución de las analogías en los libros de texto. Del mismo modo, los autores (Femández et al. 2005b) presentan los procesos analógico de enseñanza aprendizaje y describen el ACA (Modelo de Aprendizaje con Analogías) haciendo una referencia a como están ubicadas y presentadas las analogías en los libros de ciencias. Las analogías que se han identificado se encuentran localizadas en el desarrollo de la unidad didáctica o lección, se presentan en formato pictórico o pictórico-verbal. Este hecho confiere a dichas analogías un carácter secundario. 
Igualmente, Femández et al. (2001) realizan una experiencia piloto en un curso de $3^{\circ}$ de la E.S.O. encontrando una distribución de los estudiantes/as por el incremento en la nota, tanto positivo como negativo. Se seleccionaron al azar dos estudiantes de cada grupo a ambos lados de la media de la distribución de resultado. Para verificar la efectividad del aprendizaje analógico se usó una entrevista semiestructurada, después del análisis de los datos recogidos los autores dan las siguientes recomendaciones metodológicas para el estudio de la efectividad de las analogías en la dase de ciencias. En un primer lugar, la grabación de la clase en vídeo, como registro, para poder estudiar la presentación de la analogía a los estudiantes, y así observar si la analogía fue suministrada adecuadamente por el profesor(a), y si se diseñó adecuadamente el análogo. En segundo lugar, verificar que realmente se produzca un análisis de la efectividad del proceso de aprendizaje analógico, procurando que este no se reduzca a la prueba sobre una pregunta en un arestionario de una entrevista, sino en distintas pruebas, con diferentes formatos; que deben de ser debidamente contrastadas; además de estos de los hallazgos realizados en la investigación se muestran unos resultados casi iguales del aprendizaje de conceptos en los dos grupos; lo cual lleva a concluir que una analogía puede ayudar a resolver ciertas cuestiones bien delimitadas. No se puede pretender que lo resuelva todo y además es necesario utilizarlas con mucha precaución. Este estudio fue el precedente para que Elórtegui, González y Moreno (2004) posteriormente conduyeran que es muy conveniente un nuevo diseño y estudio de las analogías antes de aplicarlas con los estudiantes.

Tal como se encuentran el los libros de texto y se usan en el aula hay muy poco control sobre la forma en que se incorporan a las estructuras mentales de nuestros estudiantes, produciéndose aprendizajes erróneos simultáneamente a los correctos y omitiéndose aspectos de aprendizaje muy deseable, lo que lleva a aprendizajes incompletos. Por esto, los autores proponen iniciar el estudio de un método para el "aprendizaje con analogías" que al ser aplicado resulte más idóneo. Este método debe tener al menos, las siguientes características: primero utilizar esquemas de las analogías que describan explícitamente el tópico, el análogo y la trama de relaciones entre ellos. Segundo, explicitar las limitaciones de la analogía y enriquecer la trama de relaciones más allá de las semejanzas superficiales. Tercero, basarse en materiales con una presentación asequible para el profesorado en general, sin induir tecnicismos innecesarios pero manteniendo el rigor. Posterior a este estudio Femández et al. (2005) realizan otra revisión sobre este estudio y ponen de manifiesto hasta qué punto las previsiones de los docentes se ven desbordadas por la imaginación y la diversidad de los esquemas mentales de los estudiantes. Para ello, se utilizó como referencia sus conocimientos y la descripción del modelo, en texto e imagen, contenida en el libro de texto. Luego se utilizó una plantilla simple en forma de tabla en la que se recoge "en qué se parecen" y "en qué se diferencian" (componentes y atributos), "qué funciona igual" y "qué funciona distinto" (nexos). Este nuevo análisis da como resultado que al trabajar con constructos, jugando a las diferencias, y aplicarlo a atributos, nexos y relaciones permitía que nuestros estudiantes obtuvieran observaciones y condusiones que iban mucho más allá de las que una explicación o un libro de texto les pudieran suministrar en una actividad tradicional.

Aragón et al. (2005) analiza las relaciones existentes entre la comprensión de 65 estudiantes de tres grupos de de $3^{\circ}$ de ESO sobre las analogías trabajadas en clase. Después del análisis se halla que la comprensión de analogías influyó positivamente en la resolución de tareas en tomo al modelo cinético-molecular. Esto podría sugerir que, cuando las analogías empleadas son adecuadamente interpretadas por los alumnos, pueden contribuir a que los modelos mentales evocados como respuesta a las tareas que se les plantea, sean más adecuados desde el punto de vista cientúfico. Además, afirma que el primer modelo desarrollado se convierte en una especie de hipótesis de trabajo que puede cambiar o evolucionar con el tiempo; así se expresa la conveniencia de adoptar más de una analogía para propiciar que el modelo mental que se construya sea próximo al modelo deseable desde el punto de vista de la ciencia escolar. Así mismo, parece aconsejable el uso de la misma analogía a lo largo de distintos momentos de la secuencia didáctica, con objeto de mostrar la utilidad de la misma. Después de este estudio Oliva y Aragón (2007) nos presentan 
algunos aspectos más que en un primer aspecto interpretar los mecanismos que operan en el pensamiento analógico en la clase de ciencias, adicional a esto el grado de comprensión alcanzado por los alumnos acerca de las analogías empleadas en dase sobre el modelo cinético-molecular de la materia, y finalmente, se estudia la relación existente entre pensamiento analógico y los modelos mentales de los alumnos sobre la estructura de la materia, en el estudio se hace notorio el nivel de comprensión alcanzado por los alumnos sobre las analogías manejadas durante el proceso de enseñanza fue sólo moderado. Se crea entonces la necesidad de revisar los materiales empleados, así como la gestión didáctica que se hace de ellos. Además, el nivel de comprensión alcanzado por los alumnos sobre las analogías manejadas durante el proceso de enseñanza fue sólo moderado surge la necesidad de revisar los materiales empleados, así como la gestión didáctica que se hizo de ellos. Así mismo, la comprensión de la analogía puede facilitar a los alumnos, en los primeros estadios de la intervención, la comprensión de algunos elementos básicos del modelo cinéticomolecular, que dependerá a su vez del modelo de materia que se tenga en mente y que sirva para generar la analogía. Para terminar se hace necesario continuar evaluando el diseño de intervención mediante analogías expuesto en éste y en trabajos anteriores, a través de la elaboración de nuevos materiales que incorporen de un modo más sistemático las bases propuestas para el mismo, y mediante el diseño de didácticos de enseñanza concretos para otros temas del currículo de ciencias.

\section{Modelos didácticos para la enseñanza de las analogías}

Uno de las mayores avances en la comprensión de la estructura y descripción del modelos para el trabajo y uso de las analogías lo ha realizado el Grupo de Investigación en la Teoría de la Enseñanza y su Práctica (GITEP), quienes plantean en un estudio (2006) una primera revisión bibliográfica de las analogías más estudiadas en las dases de ciencias y el papel que estas desempeñan en la construcción del conocimiento cientúfico y su posible incidencia en el proceso de enseñanza/aprendizaje de las ciencias. El análisis de estas los llevan a plantear tres proyectos de investigación, el primero estudió las analogías presentes en los libros de texto del Área de Ciencias de la Naturaleza: tipos de analogías, idoneidad de acuerdo con los conceptos que se pretenden enseñar e idoneidad con el tipo de estudiantes a los que van dirigidas. Otro estudio que dio cuenta del campo de las analogías que usa el profesorado de ciencias en el aula al analizar si las motivaciones, metas y mundo vivencial de los estudiantes también son tenidos en cuenta. Además plantean también la necesidad de un estudio de la contribución de las analogías al proceso de enseñanza/aprendizaje: refuerzo del conocimiento de conceptos cientúficos, cambio conceptual, creatividad, estética, actitudes positivas, valores afectivos, conexiones C.T.S.

De otro lado, Femández et al. (2004) propone de una manera condensada cómo las analogías han contribuido a la construcción y desarrollo del conocimiento cientúfico y a su posterior comunicación, por lo que se consideran fundamentales en el proceso enseñanza/aprendizaje de las ciencias. El estudio también plantea una contextualización permitiendo establecer un posicionamiento acerca de la concepción y estructura de la analogía, el proceso analógico de enseñanza/aprendizaje, y los criterios que permiten dasificar las analogías presentes en los libros de textos. Además, presenta un diseño sobre un método de aprendizaje con analogías también retomado en el marco, sobre esta disertación se considera que es fundamental conocer qué concibe como analogía el profesorado y el alumnado, disponer de una recopilación y censo de las analogías que se utilizan. Se considera muy útil la elaboración de un catálogo de analogías de interés docente, hacer propuestas elaboradas didácticamente de las analogías detectadas, es apropiada una investigación orientada en averiguar cómo construye el alumnado las analogías en su proceso de aprendizaje y conocer qué procesos mentales emplea.

Posteriormente, los autores Medina et al. (2004) se interesan en como las analogías afectan e intervienen en la enseñanza/aprendizaje y circulación de las ciencias, así como, en el diseño un 
modelo de aprendizaje por analogías para identificar y analizar las analogías presentes en los libros de texto de Enseñanza Secundaria. En este estudio, se han preocupado por identificarlos, dasificarlos, y analizar las analogías presentes en los libros de texto de Enseñanza Secundaria de las editoriales de mayor difusión en España. En consequencia con lo anterior, se catalogaron veinte analogías que en su gran mayoría están relacionadas con dos grandes núcleos conceptuales: el auerpo humano y biología Celular, después de describir y enseñar la correcta estructura de dos de las veinte analogías, los autores reportan que la elaboración de un catálogo constituye un primer paso en la realización de un análisis detallado de la relación analógica, tal como propone, para posteriormente, poder profundizar en la adecuación de la analogía para facilitar el aprendizaje del tópico de enseñanza en el alumnado al que se dirige, en la forma en la que se suele encontrar la analogía y el modo en el que ésta debe presentarse en la interpretación de la analogía por los docentes y el alumnado; es decir, ccómo la entienden los profesores? y ccómo la explican los estudiantes?, y en las estrategias para un uso efectivo de las analogías en la práctica de aula.

A continuación Femández et al. (2004), realizan este nuevo estudio que es bastante interesante y en el que utilizan como recurso metodológico el crecimiento de una enredadera en analogía con los procesos de aprendizaje. La primera aplicación de esta analogía se realizó con estudiantes del aurso de Didáctica de las Ciencias Experimentales. De esta experiencia es conduyente la forma en que se propone una estructura de las analogías, modelos muy frecuentes en la enseñanza de las Ciencias, y una forma de sistematizar su estudio. La aplicación al caso de una enredadera, como comparación con el aprendizaje de los estudiantes, permite poner de manifiesto su interés metodológico en la docencia. El estudio generalizado a gran cantidad de analogías de uso habitual, permite detectar la utilidad de la propuesta como recurso metodológico en las dases diarias.

Benigno Martín González (2005) en este estudio el autor realiza una completa descripción de la forma de cómo se genera y la interacción que debeńa realiza en la estructura cognitiva para la enseñanza/aprendizaje con analogías, y así mismo, plantea el modelo ACA (Aprendizaje Con Analogías) y consiste en las tres fases siguientes a) Diseño del análogo. b) Proceso de enseñanzaaprendizaje de la analogía. c) Evaluación del proceso de enseñanza-aprendizaje, fasces que han sido explicadas y recogidas en el marco teónico.

Los estudios anteriormente reportados, son solo una muestra de los trabajos que se vienen realizando en la línea de investigación sobre el uso de las analogías en los últimos diez años, nos es posible evidenciar una evolución en esta línea pero aún queda abundantes aspectos que requieren retratados en investigaciones que sean diseñadas con este fin.

\section{Conclusión}

A pesar de lo expresado en los párrafos anteriores se hace evidente que las explicaciones por medio de analogías y su uso no es una tarea sencilla. Al ser un recurso metodológico de enseñanza y aprendizaje, va a estar impregnada de la concepción que se tiene sobre la enseñanza y el aprendizaje en educación.

Tras haber analizado algunos de los estudios que se han realizado con respecto al uso de analogías en la enseñanza y aprendizaje de las ciencias experimentales, podemos visualizar diversas tendencias de las investigaciones como lo son aspectos ya mencionados. A pesar de los avances reportados en estas líneas se hace evidente la necesidad de nuevas investigaciones, para los siguientes aspectos:

> Si las analogías son consideradas una herramienta didáctica, se hace necesario estudiar a fondo las relaciones existentes en las creencias, las formas de pensamiento y los hábitos metodológicos del profesorado. 
> Mostrar a los estudiantes los razonamientos analógicos que los científicos han llevado a cabo para la construcción de sus teorías, puede favorecer el aprendizaje, pero necesita estudios que comprueben su efectividad como estrategia de aprendizaje.

$>$ Se requiere realizar estudios que comprueben más a fondo la efectividad los modelos analógicos propuestos por los distinto autores

> Es demandante la necesidad de realizar una propuesta crítica de un catálogo de analogías de interés, que brinde analogías correctamente estructuradas y delimitadas.

$>$ Se hace necesario indagar que entienden tanto los profesores como los alumnos por analogía, y que uso hacen de esta

> Es necesario evidenciar si a través de las analogías es posible que evolucionen los modelos mentales de una manera efectiva.

$>$ Generar un conjunto de características que posibiliten el análisis, la evaluación y el diseño de la presentación de las analogías en la enseñanza de las ciencias experimentales

$>$ Establecer estrategias de enseñanza con analogías que permita identificar las concepciones previas del análogo.

$>$ Diseñar estrategias más efectivas para la integración de las TIC y el uso de las analogías.

Finalmente, y como una perspectiva para el trabajo en esta línea de investigación se plantea que concibiendo el nivel de desarrollo profesional del profesorado, en cuanto al uso de las analogías, su pensamiento se puede catalogar en tres niveles:

I. El primero, se da cuando se lograra hacer un uso consciente de las analogías.

II. El segundo, si el profesor hace un uso consciente y, además, logrará enriquecer y delimitar las analogías.

III: El tercer nivel se alcanza si el profesor no solo cumpla con los dos anteriores, si no que también lograr la interacción con el alumno, para que este participe en la creación de la analogía.

Por otro lado, y directamente relacionado con la concepción de los profesores sobre el uso de las analogías, es necesario identificar en el discurso y la práctica diaria de los maestros, la forma como estos materializan en el aula este recurso, facilitando en el alumnado la evolución de sus modelos mentales comenzando en diferentes niveles que puedan:

I. Lograr que los estudiantes expliquen el tópico puntual tratado con la analogía II. Lograr que los estudiantes puedan explicar los fenómenos derivados del tópico III.Lograr que los estudiantes realicen el proceso de modelización del conocimiento.

\section{Bibliografía}

Femández, J.; González, B.; Moreno, T. (2003). Las analogías como modelo y como recurso en la enseñanza de las ciencias. Alambique, 35, 82-89

Femández, J.; González, B.M.; Moreno, T. (2005). Hacia una evolución de la concepción de analogía. Enseñanza de las Ciencias. 23(1), 33-46.

González González, B. M. y T. Moreno Jiménez (1998). Las analogías en la enseñanza de las Ciencias. Actas II Simposio sobre la Docencia de las Ciencias Experimentales en la Enseñanza Secundaria, 204-206.

Oliva, J. Ma y Aragón, Ma.M. (2007). pensamiento analógico y construcción de un modelo molecular para la materia. Revista Eureka. Enseñanza Divulgación de las Cieniencias, 4(1), 21-41

Oliva, José Ma , Azcárate P., Navarrete A. (2007). Teaching Models in the Use of Analogies as a Resource in the Science ClassroomIntemational. Joumal of Science Education. 29(1), 45-66 